\title{
Proton microbeam irradiation effects on PtBA polymer
}

\author{
J KAMILA ${ }^{\dagger}$, S ROY, K BHATTACHARJEE, B ROUT ${ }^{\dagger \dagger}$, B N DEV, R GUICO ${ }^{\S}$, J WANG ${ }^{\S}$, \\ A W HABERL ${ }^{\dagger \dagger}$, P AYYUB ${ }^{\#}$ and P V SATYAM* \\ Institute of Physics, Sachivalaya Marg, Bhubaneswar 751 005, India \\ ${ }^{\dagger}$ Department of Physics, B.J.B. Junior College, Bhubaneswar 751 014, India \\ ${ }^{\dagger}$ Louisiana Accelerator Centre, PO Box 42410, Layette, LA-70504, USA \\ ${ }^{\dagger \dagger}$ Department of Physics, University at Albany, Albany, NY 12222, USA \\ ${ }^{\S}$ Advance Photon Source, Argonne National Laboratory, Argonne, IL-60439, USA \\ \#DCMP\&MS, Tata Institute of Fundamental Research, Mumbai 400 005, India
}

MS received 13 November 2004

\begin{abstract}
Proton beam lithography has made it possible to make various types of 3D-structures in polymers. Usually PMMA, SU-8, PS polymers have been used as resist materials for lithographic purpose. Microbeam irradiation effects on poly-tert-butyl-acrylate (PtBA) polymer using 2.0 MeV proton microbeam are reported. Preliminary results on pattern formation on PtBA are carried out as a function of fluence. After writing the pattern, a thin layer of Ge is deposited. Distribution of Ge in pristine and ion beam patterned surface of PtBA polymer is studied using the optical and secondary electron microscopic experimental methods.
\end{abstract}

Keywords. Ion micro-beam; pattern generation; PtBA polymer; Ge on polymers.

\section{Introduction}

Recently, the fabrication of sensor and actuator devices on the micro-scale and their integration with electron devices and micro-electromechanical systems (MEMS) has gained a highly technological importance (Ikuta and Hirowatari 1993). With the advent of nanoscience, nano-electromechanical systems (NEMS) show promising future (Roukes 2001). The majority of sub-micron machining procedures involve two types of interactions with the resist materials: electromagnetic radiation (e.g. optical, UV or X-ray photons) or charged particles (electrons, low energy heavy ions, high energy light ions). In general, the micromachining procedures based on electromagnetic radiation require masks. The use of mask with the charged particles is not convenient, as the greater energy deposited in the mask during exposure results in mask instabilities due to heat expansion, stress and damage. Thus, the ion beam micromachining is limited to direct writing processes (Breese et al 1996). The photolithography utilizes optical light passing through a patterned mask. In the photolithography, the transmission of light exposes a specific geometrical pattern in a resist layer deposited on a substrate and depending on the positive or negative type of resist material, the exposed or unexposed part gets chemically etched out forming the microstructure. Although photolithography is essentially a surface micromachining technique and therefore two-dimensional,

*Author for correspondence (satyam@iopb.res.in) various wet and dry etching techniques have been successful in producing 3-D microstructures. X-ray lithography has the capability of manufacturing 3-D structures with high aspect ratios because of the penetration properties of X-rays in resists such as polymethylmethacrylate (PMMA). When the developed resist is used in conjunction with electro-deposition, which fills the resist mold with metal, then 3-D metallic microstructures can be produced. This process is now universally known by its German acronym LIGA. The use of LIGA is limited for mass production as it requires higher photon flux using synchrotron radiation sources (Rogner et al 1992; Patenburg and Mohr 2001; Madou 2002).

Among the high energy charged particle lithography, proton beam micromachining (PBM) [or deep ion beam lithography (DIBL)] is a well known method (Breese et al 1996; Watt 1999; Gonin and Munnik 2003). PBM uses energetic proton beams (few $\mathrm{MeV}$ energy) for patterning in resist materials. It is advantageous to use PBM compared to other 3-D lithographic techniques as it is a direct writing process (i.e. no masks are required), control in construction of slots, channels, holes, complex non-prismatic shapes, etc (Watt 1999; Gonin and Munnik 2003). But the PBM process is slow for commercial direct high volume batch production of microcomponents. The use of quadrupole lenses to obtain a finely focussed beam spot coupled with the ability to scan the beam in complicated patterns has resulted in microstructures of intricate 3D shapes and smooth side walls (Watt 1999; Gonin and Munnik 2003). 
Study of ion induced modification in polymers has been an active area for last two decades (Hioki et al 1983; Rao and Lee 1996). A basic understanding of physical and chemical modifications enables the use of polymers in lithography, preparation of filters, window materials for external ion beam applications such as external particle induced X-ray emission (PIXE), etc. Ion irradiation of polymers can induce irreversible changes in their macroscopic properties such as electrical and optical properties (Hioki et al 1983) and mechanical properties (Rao and Lee 1996). Electronic excitation, ionization, chain scission and cross-links as well as mass losses are accepted as the fundamental events that led to the observed macroscopic changes (Abel et al 1995). It is known that high energy ion-induced effects result in track formation in polymer (Bouffard et al 1995). Recently, various groups at the Nuclear Science Centre, Delhi, had been working on various aspects of modification in polymers, like track formation, hydrogen loss, etc using swift heavy ions (Avasthi et al 1998; Rao et al 2003; Mujahid et al 2004). Zhu et al (2002) concluded that not only the physical processes but also the chemical processes of the energy deposition determine the modification of polymer.

For lithographic purpose, various types of resist materials have been used. Among them, PMMA and SU-8 were successfully used for PBM. While PMMA is a positive type of resist, the SU- 8 is a negative type. $\mathrm{Su}-8$ requires relatively less dose but the aspect ratios obtained are smaller compared to those obtained using PMMA (van Kan et al 1999). In this article, we present preliminary results using Institute of Physics microbeam facility for the purpose of patterning with a radiation damage on PtBA polymer. Poly(tert-butyl acrylate) (PtBA) is an amorphous homopolymer. This polymer is found to be resistant to photooxidation and has a glass transition temperature, $\approx 49^{\circ} \mathrm{C}$ (Ahn and Shull 1996; Richter et al 2001).

With the impact of progress in nanoscience, active research has started on the 'bottom-up' approach. The 'bottom-up' approach could enable to build nanodevices with an atom or molecule as a base unit. Feynman gave a visionary talk in 1959 titled 'there is plenty of room at the bottom' in which he essentially argued for 'bottom-up' approach (http). The other approach, 'top-down' has been the basis for integrated circuit technology in the past. The two approaches, the 'top-down' and the 'bottom-up' needed to be efficiently combined to realize nanostructures for technological applications. Growth of various semiconducting and metal nanoparticles on a polymer follow the 'bottomup' approach as the self-assembly dominates. But in this type of self-assembly, in general, in all self-assembly processes, there is no control over the position or placement. To control the placement of the nanoparticles following self-assembly, one requires a sort of pre-patterning. Das et al $(2000,2001)$ proposed the possibility of growth of one or two-dimensional lattice of Ge islands if the polymer surface is patterned. Zhong et al $(2003,2004)$ have grown a two-dimensional periodic positioning of selfassembled Ge islands on pre-patterned $\mathrm{Si}(001)$ substrates. In order to understand the growth of semiconductor particles on a patterned surface of a polymer, proton ion microbeam for patterning the polymer surface and high vacuum deposition using resistive heating has been used.

Here, we report the first use of ion-microbeam facility at the Institute of Physics, for writing a pattern on PtBA polymer and growth of $\mathrm{Ge}$ islands on the patterned polymer surface.

\section{Experimental}

An ion microbeam facility has been set up at one of the beam lines of the $3 \mathrm{MV}$ tandem Pelletron accelerator at the Institute of Physics, Bhubaneswar, in collaboration with the University at Albany, USA (Rout 2001; Rout et al 2001). A spatial resolution of $\approx 3 \mu \mathrm{m}$ with a beam current of about $80 \mathrm{pA}$ with a $3 \mathrm{MeV} \mathrm{He}^{+}$beam was achieved. Figure 1 schematically shows the microbeam facility. A set of micropolished slits and a powerful magnetic quadrupole doublet lens are used. The electrically isolated pole pieces of the lens are used as deflection plates to scan the beam across the sample. A motorized $\mathrm{X}-\mathrm{Y}$ stage is used to position the sample. The target chamber houses three detectors: a cooled $\mathrm{Si}(\mathrm{Li})$ detector used to analyse X-rays, a surface barrier detector to analyse backscattered ions and an aluminized plastic scintillator for detecting secondary electrons from the target. A high resolution CCTV camera-microscope along with light guiding system helps to view the target optically. The system is equipped with a rear microscope for directly looking at the beam spot. The necessary hardware and software have been developed for control of the beam, data acquisition and autopumping/venting of the target chamber. Both RBS and PIXE data are collected, stored and displayed simultaneously through the attached computer. The same computer is also used to store the digitized image of the sample in the SEM mode and to control the X-Y stage for the sample holder. A representative pattern 'IOP' is depicted in the inset of figure 1 . The position coordinates of 'IOP' is written in a file with $4096 \times 4096$ pixels depth, which eventually gets converted to respective voltages in $\mathrm{X}$ and $\mathrm{Y}$ scanners by the program for 2-D beam scanning. To scan the beam in a desired pattern one needs to give the set of $\mathrm{X}, \mathrm{Y}$ coordinates to the program which then converts to proportional analog voltages and supplies to the $\mathrm{X}$ - and Y-deflectors to deflect the beam in a programmed fashion. The pattern is generated by writing $\mathrm{X}, \mathrm{Y}$, and $\mathrm{Z}$ coordinates in a text which is read by the program. The Z-coordinate corresponds to time for which the beam will stay at a given $\mathrm{X}, \mathrm{Y}$ coordinate. At present, two modes of operation are available: continuous mode and point mode. In continuous mode, the beam position can be varied continuously. In point mode the beam stays at a position for 


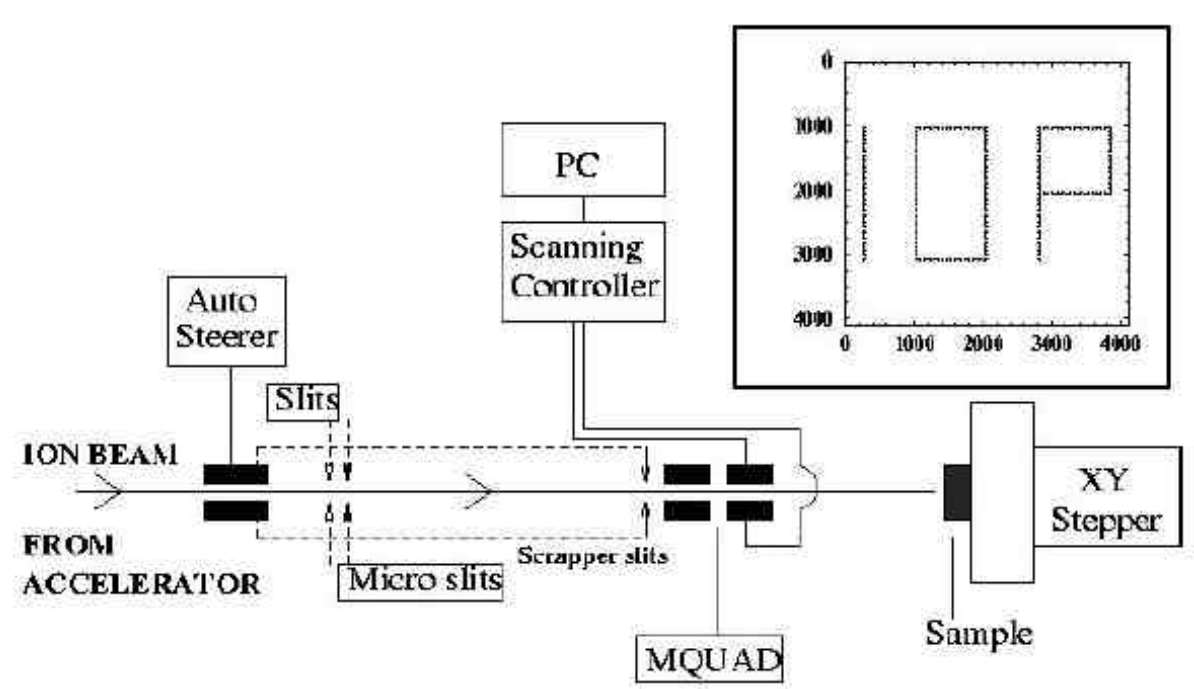

Figure 1. Schematic set up of ion-microbeam facility at the Institute of Physics and inset shows the position coordinates that are used to write 'IOP'.

a pre-set time and then moves to the next point. This will make the program more versatile as one can pattern in $\mathrm{Z}$ coordinate (which corresponds to more damage) also depending on the time at different points. For the present work we used the beam in continuous mode. Thus to begin with we wrote the $\mathrm{X}, \mathrm{Y}$ coordinates in $4096 \times 4096$ pixels for 'IOP' which is shown in the inset of figure 1 . Present writing program takes the top left corner as the origin.

Poly(tert-butyl acrylate) (PtBA) with a molecular weight of 3,01,000 was synthesized by anionic polymerization at $-78^{\circ} \mathrm{C}$ in tetrahydrofuran as described in Ahn and Shull (1996). The PtBA thin films were prepared by spin coating using solutions of PtBA in butanol. The silicon substrates were cleaned prior to deposition. A $100 \mathrm{~nm}$ thick PtBA film was then coated at $2000 \mathrm{rpm}$. A $2 \mathrm{MeV}$ proton beam was used for pattern writing. This beam can scan $250 \times 250 \mu \mathrm{m}^{2}$ area with the existing set up. As the purpose of the present study is to make a pattern (radiation damaged area) and then deposit Ge islands on the patterned surface, a beam size of $\sim 10 \mu \mathrm{m}$ was used. A direct measurement of total fluence was not possible and hence fluence has been controlled with the scan time. Scanning electron microscopic measurements were carried out at TIFR, Mumbai.

\section{Results and discussion}

$\mathrm{MeV}$ ions passing through polymer films modify their electrical and optical properties and these changes are related to changes in the chemical structures of the polymer. The penetration depth of proton beam in polymers, in general, is much more than the thickness of the PtBA film (e.g. $2 \mathrm{MeV}$ proton penetrates $62 \mu \mathrm{m}$ into PMMA). Figures 2 and 3 show optical micrographs after irradia-

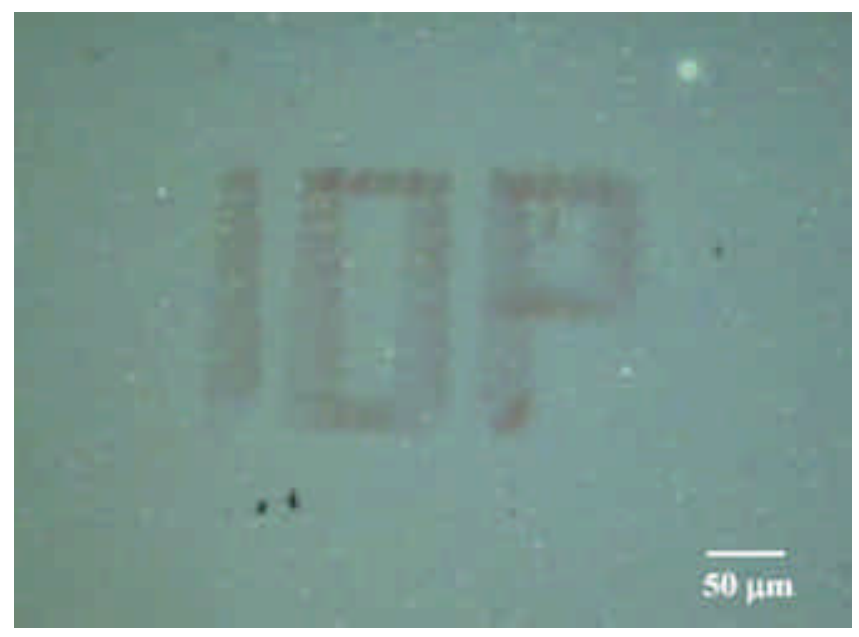

Figure 2. Optical micrograph of 'IOP' patterned on PtBA.

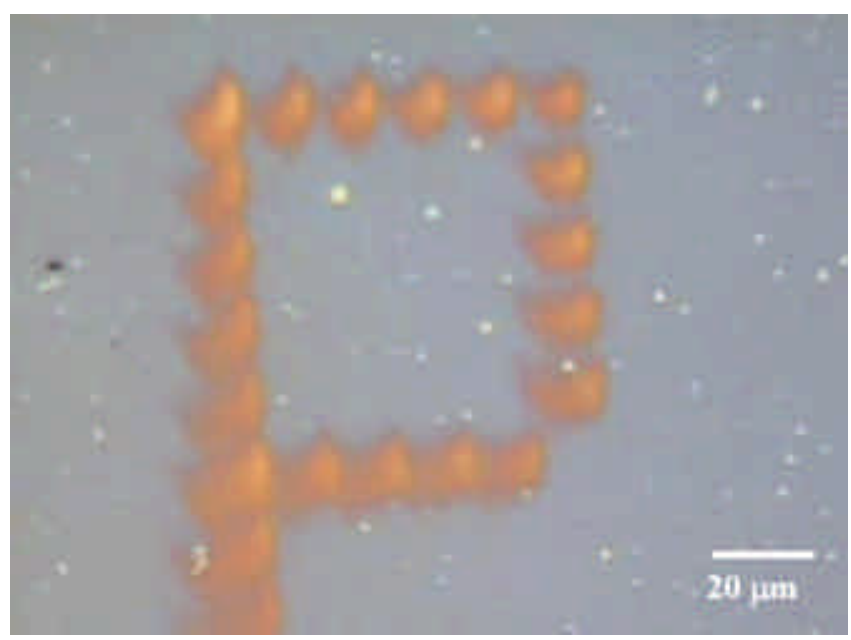

Figure 3. Optical micrograph of ' $\mathrm{P}$ ' patterned on PtBA for effective longer exposure. 


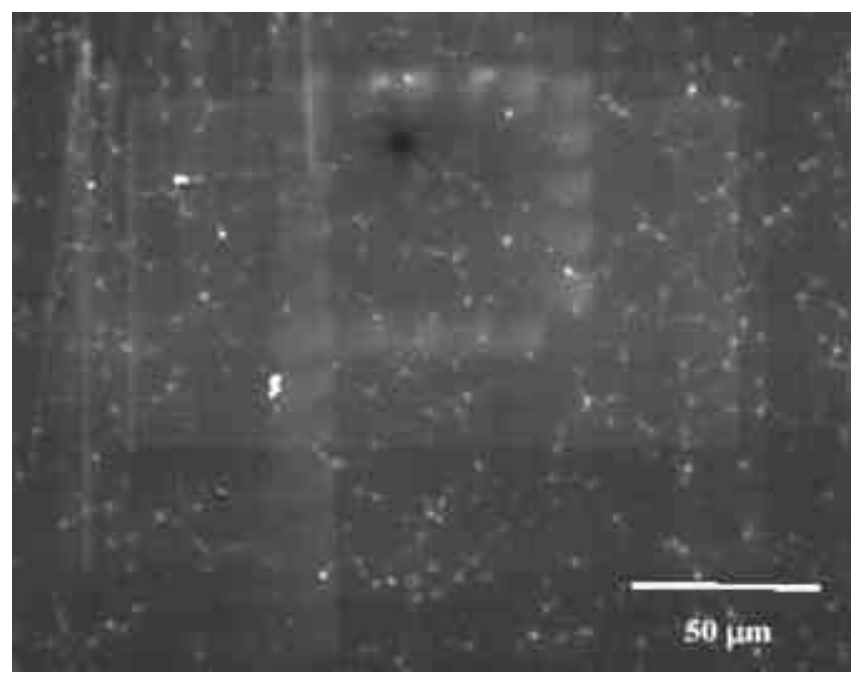

Figure 4. SEM micrograph of Ge $(\approx 10 \mathrm{~nm}$ thick) deposition on PtBA surface after ion beam writing.

tion. The ion beam typically of $10 \mu \mathrm{m}$ size has been programmed to scan in the pattern 'IOP' for about $2.5 \mathrm{~h}$. We could not measure the current of the beam and also the total dose. At constant source current of $\approx 1.0 \mu$ (typically, this results in $\approx 50 \mathrm{pA}$ at the target in $\approx 3 \mu \mathrm{m}$ spot size), irradiation time was varied to change the total fluence on the sample. The optical micrograph in figure 2 shows the pattern 'IOP' with contrast in colour. A scan for $2.5 \mathrm{~h}$ irradiation time is used for this purpose. The optical properties change and hence the ion-irradiated location has a different colour. From figure 2, it is evident that our facility can be used in principle for microbeam patterning applications. A scan for $0.5 \mathrm{~h}$ irradiation time under similar conditions showed no change in colour at the irradiated part of the polymer. It appears that a threshold fluence might be present for change in colour to be observed. To have larger fluence, for total $2.5 \mathrm{~h}$ of irradiation, writing has been carried out only for ' $\mathrm{P}$ ' instead of 'IOP'. Figure 3 shows an optical image of ' $\mathrm{P}$ '. The contrast for ' $\mathrm{P}$ ' is better than that of 'IOP' due to more time of exposure. Our next step is to look for any preference in the growth of $\mathrm{Ge}$ on the patterned polymer surface.

Ge nanoparticles have been grown usually on silicon substrates by molecular-beam epitaxy (MBE) and by other methods. The lattice mismatch between $\mathrm{Ge}$ and $\mathrm{Si}$ and the consequent strain leads to a layer-plus-island growth process (known as Stranski-Krastanow (SK) grow mode) in which Ge islands grow on a uniform Ge layer. In the SK mode growth, it is not possible to grow isolated Ge quantum dots. Using other methods, isolated self-assembled $\mathrm{Ge}$ nanostructures have been grown on polymers by thermal evaporation under high vacuum conditions utilizing the non-wetting condition given by the surface energy relation: $\sigma_{\mathrm{Ge}} \gg \sigma_{\text {polymer }}$ (Das et al 2000, 2001). In these studies, self-assembled nanostructural islands, isolated or coalesced on the line defects into lattice-like or continuous structure have been observed to form at nominal film thickness $(\approx 5 \mathrm{~nm})$ of $\mathrm{Ge}$ on $35 \mathrm{~nm}$ thick polymer blend (15\% polystyrene (PS), $85 \%$ poly( $p$-bromostyrene-stastyrene) $\left.\left(\mathrm{PBr}_{0.06} \mathrm{~S}\right)\right)$ (Das et al 2000, 2001). Growth pattern varied from isolated islands of $\mathrm{Ge}$ to wires of $\mathrm{Ge}$ as the Ge film thickness was increased on PtBA polymer surfaces. Isolated $\mathrm{Ge}$ islands were found for a $\mathrm{Ge}$ thickness of $\leq 0.9 \mathrm{~nm}$ on the PtBA polymer surface (Satyam et al 2005). The change of growth pattern on the irradiated portion of the specimen will be an interesting issue. After irradiating in a programmed manner (' $P$ ' surface), a $10 \mathrm{~nm}$ thick Ge is deposited on the patterned PtBA surface. Figure 4 shows a SEM micrograph following Ge deposition. The energy dispersive X-ray spectrometry data (EDS) do not show any preferential growth of Ge in and around the irradiated part. Though the preferential growth of $\mathrm{Ge}$ is absent, it is required to do more precise measurements with nano-sized beams for patterning and smaller thickness of $\mathrm{Ge}$ on the nano-patterns and better imaging is necessary to understand this particular problem.

\section{Conclusions}

We have demonstrated the first use and capability of pattern writing using the ion microbeam facility at the Institute of Physics, Bhubaneswar. The patterned damage on PtBA polymer caused due to $2 \cdot 0 \mathrm{MeV}$ proton beams has been used to study growth of Ge nanoislands on pre-patterned polymer surfaces.

\section{Acknowledgements}

We would like to thank the staff of IBL, Institute of Physics, for their help for carrying out this experiment. Thanks to Ken Shull at Northwestern University for providing the polymer.

\section{References}

Abel F, Quillet V and Schott M 1995 Nucl. Instrum. \& Meth. B105 86

Ahn D and Shull K 1996 Macromolecules 294381

Avasthi D K, Singh J P, Biswas A and Bose S K 1998 Nucl. Instrum. \& Meth. B146 504

Bouffard S, Gervais B and Leroy C 1995 Nucl. Instrum. \& Meth. B105 1

Breese M B H, Jamieson D N and King P J C 1996 Material analysis using a nuclear microprobe (New York: Wiley)

Das Amal K, Kamila J, Dev B N, Sundaravel B and Kuri G 2000 Appl. Phys. Lett. 77951

Das Amal K, Kamila J, Dev B N, Sundaravel B and Kuri G 2001 Appl. Phys. Lett. 783552

Gonin Yvan and Munnik Frans 2003 Appl. Surf. Sci. 217289

Hioki T, Noda S, Sugiura M, Kakeno M, Yamada K and Kawamoto J 1983 J. Appl. Phys. Lett. 4330 
http://www.its.caltech.edu/ feynman/plenty.html

Ikuta K and Hirowatari K 1993 Proceedings of IEEE international workshop on micro electro-mechanical systems (New Jersey: IEEE) pp 42-47

Madou M J 2002 Fundamentals of microfabrication (Boca Raton, FL: CRC Press) 2nd edn

Mujahid M, Singh Padam, Srivastva D S, Gupta Shiuli, Avasthi D K and Kanjilal D 2004 Rad. Meas. 38197

Patenburg F J and Mohr J 2001 Nucl. Instrum. \& Meth. A4674681269

Rao G R and Lee E H 1996 J. Mater. Res. 112661

Rao Vijayalakshmi, Amar J V, Avasthi D K and Charyulu Narayana 2003 Rad. Meas. 36585

Richter Andrew, Guico Rodney and Wang Jin 2001 Rev. Sci. Instrum. 723004

Rogner A, Eichert J, Munchmeyer D, Pertersand R P and Mohr J 1992 J. Micromech. Microengg. 2133
Roukes M L 2001 Phys. World 1425

Rout B 2001 Growth, characterization and thermal behaviour of epitaxial metallic layers on semiconductors and their selfassembled microstructures, Ph D Thesis, Utkal University, Bhubaneswar

Rout B, Ghose S K, Mahapatra D P, Dev B N, Bakhru H and Haberl A W 2001 Nucl. Instrum. \& Meth. B181 110

Satyam P V et al 2005 Physica E27 235

van Kan J A, Sanchez J L, Xu B, Osipowicz T and Watt F 1999 Nucl. Instrum. \& Meth. $\mathbf{B 1 5 8} 179$

Watt F 1999 Nucl. Instrum. \& Meth. B158 165

Zhong Zhenyang, Halilovic A, Fromherz T, Schaffler F and Bauer G 2003 Appl. Phys. Lett. 824779

Zhong Zhenyang, Halilovic A, Lichtenberger H, Schaffler F and Bauer G 2004 Physica E23 243

Zhu Zhiyong, Sun Youmei, Liu Changlong, Liu Jie and Jin Yunfan 2002 Nucl. Instrum. \& Meth. B193 271 